\title{
Patient adherence and factors influencing quality of life in the case of osteoarthritic patients
}

\author{
HELGA FEKETE ${ }^{1}$, RÓBERT FEKETE² ${ }^{2}$ ILDIKÓ CSÓKA ${ }^{1}$
}

\author{
${ }^{1}$ Institute of Pharmaceutical Technology and Regulatory Affairs, University of Szeged, Faculty of Pharmacy - Szeged, Hungary \\ ${ }^{2}$ Teaching Hospital - Bács-Kiskun County Hospital, Establishment in Kiskunfélegyháza, University of Szeged - Kiskunfélegyháza, Hungary \\ Corresponding author: Dr. Ildikó Csóka \\ E-mail: csoka@pharm.u-szeged.hu
}

Received: 10 January 2020 / Revised: 10 February 2020 / Accepted: 10 February 2020

\begin{abstract}
Introduction: 135 million humans are affected by osteoarthritis worldwide, which is going to be doubled by 2020. Current treatment options are limited and complex, requiring the active participation of the patients in order to reach the optimal therapeutic outcome. To date, there is no study available measuring the adherence and quality of life of Hungarian arthritic patients.

Aims: Evaluation of risk factors affecting quality of life and adherence in the case of Hungarian patients, in order to identify the potential intervention points.

Methods: Hospitalized patients' final reports were analyzed retrospectively (gender, age, BMI, therapy, co-morbidities).

Results: 400 cases were evaluated (females 69\%) with an average age of 72 years. $80 \%$ of the patients had abnormal BMI. Non-pharmacological treatments ensured the longest asymptomatic period, medication histories showed polypharmacy. Co-morbidities were observed in almost every case.

Conclusion: Patient centeredness is necessary, based on multidisciplinary healthcare team to support the expected quality of life.
\end{abstract}

Keywords: osteoarthritis, adherence, quality of life.

\section{Introduction}

In chronic conditions, the World Health Organization $(\mathrm{WHO})$ considers it essential to maximize the patients' willingness to co-operate with therapies; and it classified the factors influencing adherence into 5 dimensions. The study was made in 2003 and it is still regarded as a standard document by researchers studying patient adherence. With regards to the ever aging societies, like Hungary, needs to highlight the prevalence and the incidence of the chronic disorders. [1]. Among chronic conditions, diseases affecting the musculoskeletal system are on the rise; besides the basic disease, these cause several other diseases, loss of quality of life, loss of working capacity on the labour market, etc., and thus they put an increasing burden on the patients concerned and on the society both regarding quality of life and in economic terms.

The importance and the severity of the issue

List of abbreviations: Osteoarthrosis - OA; World Health Organization - WHO; National Institute for Health and Care ExcellenceNICE; Non-Steroidal Anti-Inflammatory Drug - NSAID were recognized worldwide and the 'Decade of Bones and Joints' was proclaimed in 2000, which was extended until 2020 due to its success, and several other European organizations have also taken steps to focus attention on the disease [2-4]. Hungary was the first to join the program on state level and achieved major success in many areas [5, 6]. In spite of all these efforts, very few Hungarian studies have been made and published on OA (erosion of articular cartilage).

Arthrosis is the leading cause of disability and pain all over the world and was ranked as the 6th most common cause of disability in 2003, estimated to be the 4th in rank in 2020 [7-9]. In Hungary, the number of patients affected can only be estimated; during the European Health Interview Survey of $2014,17 \%$ of the people asked were found to have arthrosis-related joint pain, which can mean the involvement of about $\sim 1,600,000$ people nationwide [10].

The measurement of the quality of life and adherence of patients suffering from OA is complicated by several factors, which are the following:

- The disease is usually diagnosed when it is already in an advanced stage, i.e. when there are severe symptoms. The therapeutic options are 
limited; what can be achieved is mainly the alleviation of the symptoms for a longer or shorter period of time [11-13].

- As opposed to other musculoskeletal disorders, no well-proved disease-modifying active ingredient is available [14, 15].

- Due to the complex nature of the disease, the patient's active and conscious cooperation with the healthcare professionals involved in the treatment is essential.

The therapy is described in the NICE guideline [16], which divides the tasks into three separate parts. The first and foremost is the education of patients during treatment, both by doctors and pharmacists, which includes the promotion of a healthy lifestyle, the incorporation of sports into everyday life, i.e. health-conscious behavior; and also adopting the so-called Mediterranean diet [17]. All these reduce the risk of the most important risk factor, obesity, as well as the development or worsening of cardiovascular diseases, diabetes mellitus and mental illnesses, which are also risk factors in the incidence of OA.

Another element of therapy is the use of nonpharmacological therapies, which include electrical impulse therapies, manual therapies and balneological therapies. Currently, these provide the longest asymptomatic period. Paracetamol and non-steroidal anti-inflammatory drugs (NSAIDs), usually applied topically then orally, are the drugs of first choice in medicated therapies. If they fail to be effective, the administration of the opioid analgesic tramadol is recommended. Most of the active ingredients mentioned in the first group are also available in drugs without a prescription, so the extent of their use cannot be measured in this disease; it is well-known that their excessive and combined use generates several adverse effects and causes additional burdens.

The protocol does not recommend the use of chondroprotective drugs, although patients often expect these "miracle drugs" to rebuild cartilage. Commercially available products are available to users through a number of distribution chains, frequently bypassing healthcare professionals; this can result in uncontrolled use if communication between the parties is inadequate during patient care.

The rational use of the great number of overthe-counter drugs and chondroprotective preparations can be controlled only by the pharmacist, who can provide information to the family doctor and to the specialist, and can also recommend the patient to visit the doctor.

On the other hand, physicians and physiotherapists can inform the pharmacist about the patients' medical history, who can then dispense the medication with this knowledge.

In the light of all these facts, it can be hypothe-

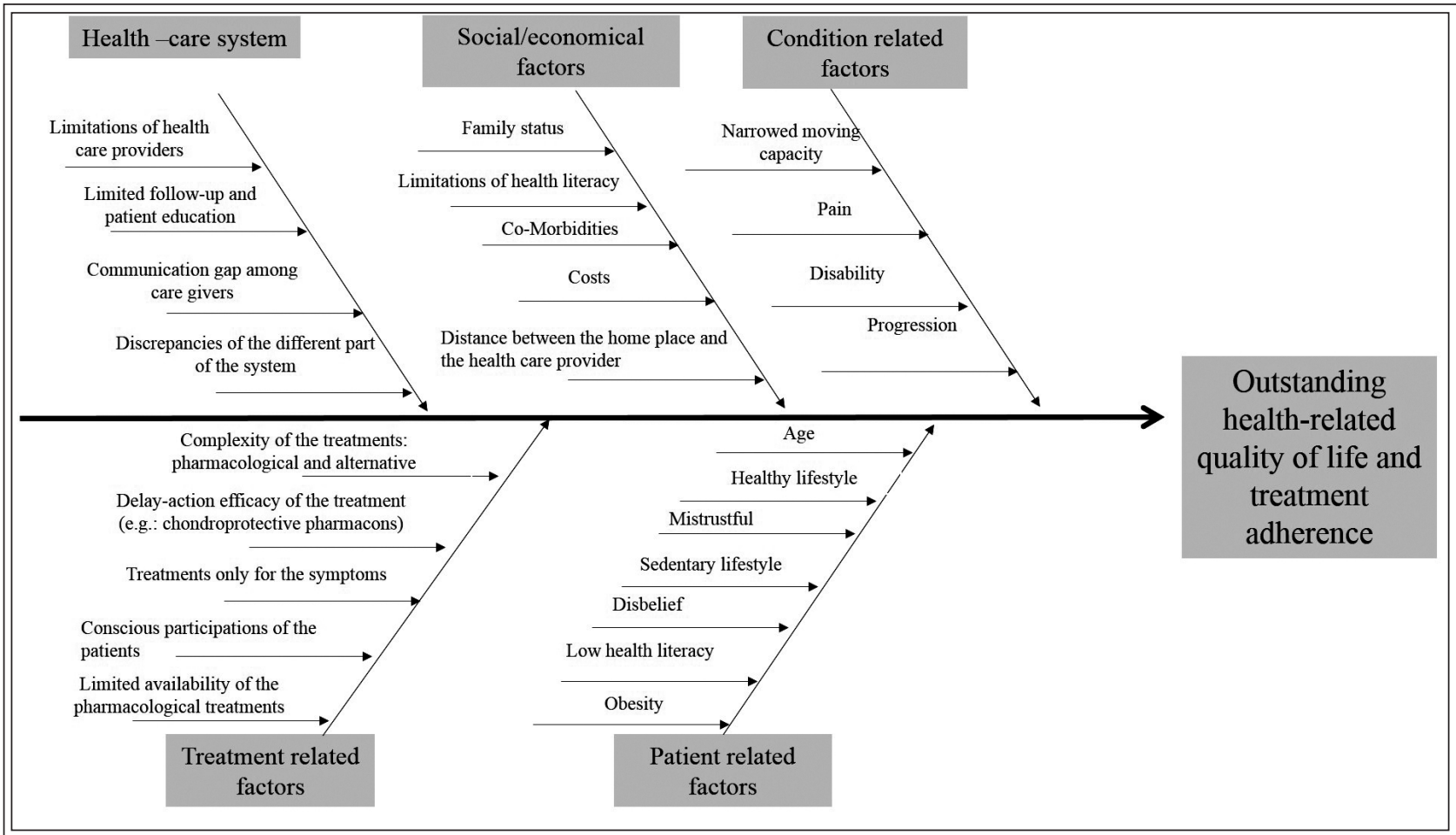

Figure 1 Division of the population in question by age and gender 
sized that adherence can be improved if OA patients' burdens affecting therapeutic cooperation are explored. With the improvement of adherence, patient satisfaction and cooperation with therapy improves, which in turn has a favourable influence on specific parameters of quality of life, resulting in improvements at individual, family and social levels. Given the complexity of the therapy, this can be achieved only through the

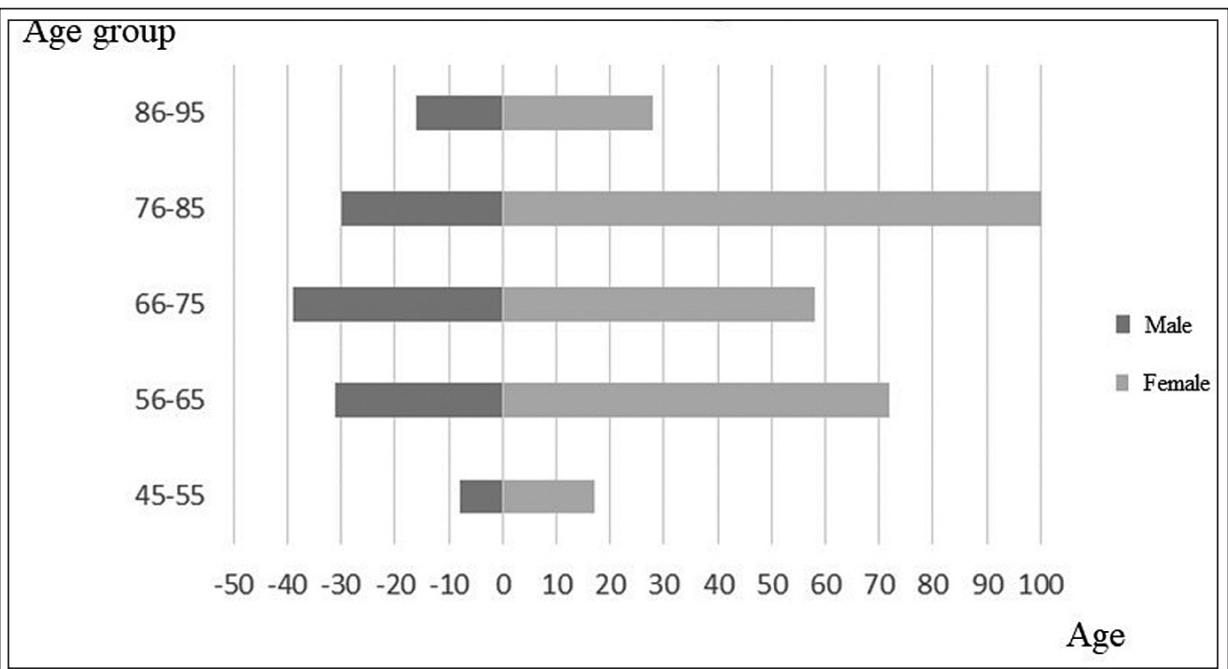

Figure 2 Cause and effects relationships between factors influencing osteoarthrosis

collaboration of the healthcare professionals participating in the treatment, which will improve therapeutic efficiency. Therefore, the aim of this survey is to explore the most common causes in order to identify potential intervention points based on the 5 dimensions of adherence of the WHO. The causal (Ishikawa) diagram (Figure 1) made on the basis of literature research illustrates the factors that influence the quality of life and cooperation of arthritic patients, which, at the same time, can also be the most important intervention points for improving the therapeutic outcome.

\section{Methods}

As the first step, the affected population was assessed retrospectively, in the course of which the data of patients treated by the Bács-Kiskun Coun-

Table I Descriptive statistics regarding to age

\begin{tabular}{l|l|c}
\hline \multicolumn{2}{l|}{ Aspects } & Values \\
\hline Number of & Valid & 400 \\
\cline { 2 - 3 } patients (N) & Missing & 0 \\
\hline Mean & 72.10 \\
\hline Median & 74.00 \\
\hline Std. Deviation & 10.81 \\
\hline Minimum & 45.00 \\
\hline Maximum & 93.00 \\
\hline
\end{tabular}

Table interpretation:

- N: sample size

- Valid: Actual number of sample size

- Missing: Missing number of sample

- Mean: Average age based on the evaulated sample

- Median: Median of the age based on the evaulated sample

- Standard Deviation (Std. Deviation): Dispersion of the age of the examined sample around the mean age

- Minimum: The lowest age in the sample tested

- Maximum: The highest age in the sample tested ty Teaching Hospital of the University of Szeged, the Musculoskeletal Rehabilitation Unit of the Establishment in Kiskunfélegyháza was processed. During the data collection period we randomly selected five anonymised patient discharge reports

Documents from the period of 2007-2013 were selected for the study; the inclusion criterion was the diagnosis of lower limb OA.

Assessment aspects:

- gender

- age

- body mass index (BMI)

- residence,

- average of nursing days,

- non-pharmacological therapies received during hospitalization, medicines received,

- existing co-morbidities.

Statistical evaluation was performed with Microsoft Excel 2013 and Version 23 of the SPSS program package.

The study was conducted with the permission of the Scientific and Research Ethics Committee, file number: 24950-3/2016/EKU.

\section{Results}

\subsection{Gender and age of patients}

A total of 400 final reports were evaluated during the study period. The majority of cases $(69 \%)$ were female with an average age of 72 years (Table I). It can be seen that the majority of patients were in the age group 76-85 for women and 66-75 for men (Figure 2). This result is not surprising as the number of patients who visit a healthcare institution 


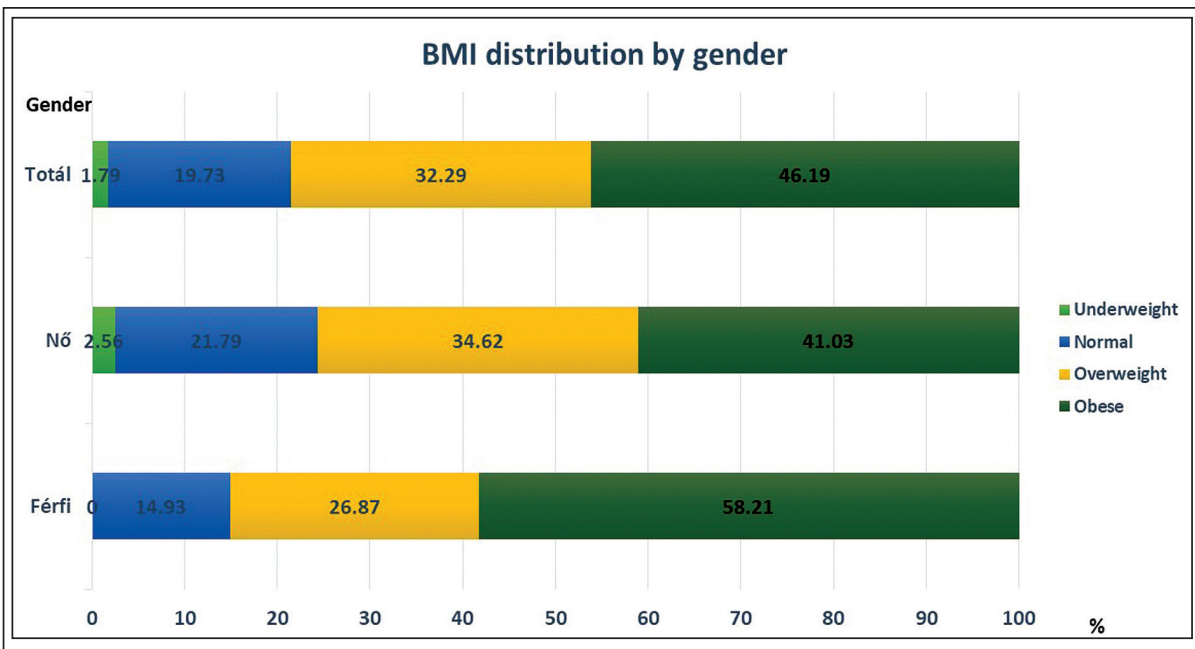

Figure 3 Division of the population in question by Body Mass Index BMI categories in accordance with WHO classification : $<18.5$ underweight, 18.51 - 24.9 normal, 25.0 -29.99 overweight, $>30.0$ obese

with complaints increases dramatically over the age of 60 . As regards age analysis, it should be noted that 128 patients $(32 \%)$ were under 65 years of age, i.e. in active working age. Considering that the average of nursing days for the sample examined was 18.6 days, this means that the affected persons were unable to work and to perform their duties minimum for this period. There was no significant relationship ( $p=0.075, \mathrm{NS})$ between gender and age at significance level 0.05

\subsection{Relationship of body mass index (BMI) with gender and age}

The most important risk factor for arthrosis is overweight. Inadequate body mass puts enormous strain on the joints and cartilage, which are eroded continuously during daily activities; and this can be further aggravated by the lack of sports activities in the daily routine.

From among the 400 cases examined, the data necessary for BMI calculation (body weight and height) were available in 224 cases. The results are summarized in Figure $3 \sim 46 \%$ of the patients were found to be in the most severe "obese" category; overall, $\sim 80 \%$ of the cases deviated from the "normal" body weight category (BMI categories, based on the classification of the WHO). There was no significant difference between men and women $(\mathrm{p}=$ 0.148 ). Such a serious weight problem not only enhances the development and progression of musculoskeletal disorders but also forms the basis of many other chronic conditions, which increase the likelihood and frequency of hospitalization as well as the number and quantity of medications taken.

\subsection{Hungarian data related to the recommendations of the} NICE disease-specific guideline

Today the treatment of arthrosis is primarily limited to relieving symptoms; the reason for this is that although the development of therapies for the modification of the course of the disease is researched extensively Currently there is no active ingredient available with an adequate level of evidence and the treatment related risk is still higher than the therapeutic benefit

For the sake of completeness, it should be noted that patient education recommended in the first place is going to be the subject of the following study; no data regarding it were available in the current retrospective survey.

\subsection{Non-pharmacological therapy}

The guideline identifies non-pharmacological therapeutic options as the level following patient education. These treatments can be received in the rehabilitation centers operating in healthcare institutions or in qualified spas, with a specialist's referral. As the studied population underwent musculoskeletal rehabilitation treatment, our study also included the evaluation of non-pharmacological treatments received during hospitalization. The guideline groups therapies according to their focus on muscle strengthening, gymnastic exercises or the use of electrical impulse. We grouped the survey data according to this, and if they did not specifically belong to any of the groups, they were classified into the so-called "other" category. The results are shown in Figure 4.

Gymnastic exercises included individual and group exercises performed on land and in water, with or without help (striped marking). Mobilization, weight bath and medicinal bath were grouped into the category of muscle strengthening (black marking); while treatments based on electrical impulse included Tangentor, galvanic treatment, iontophoresis, TENS and the use of BEMER mattress (checkered marking). Ultrasound and mud pack were classified in the "other" category (grey marking). 


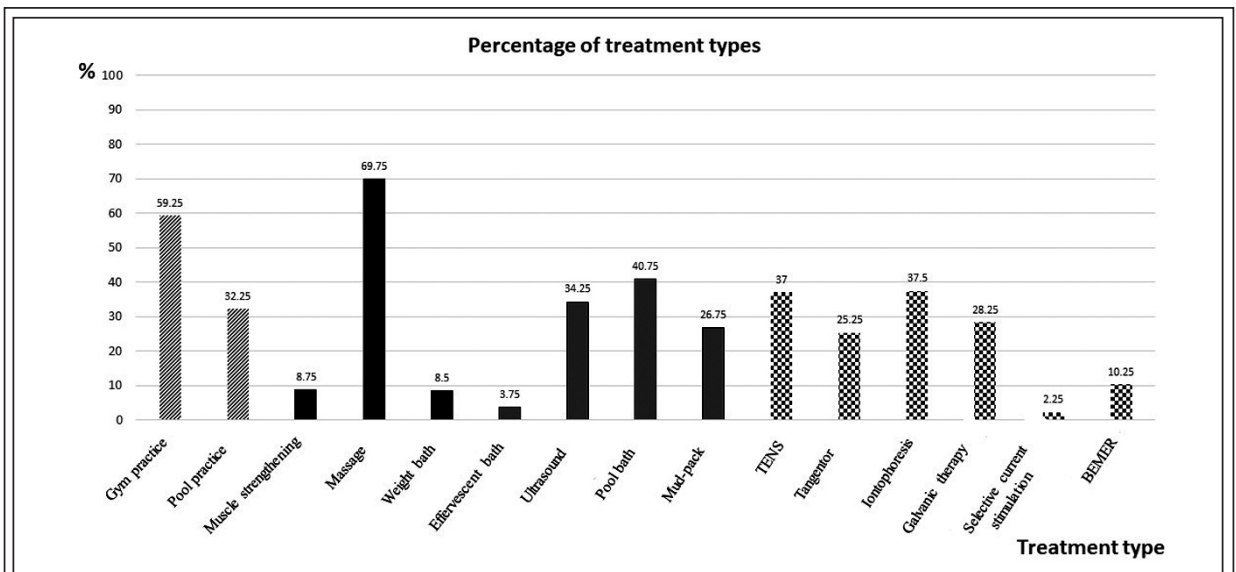

Figure 4 Percentage distribution of non-pharmacological therapies during the study period pervision. Needless to say, their regular performance requires a high degree of patient adherence.

\subsection{Evaluation of pharma- cological therapy}

As already mentioned in the introduction, the NICE guideline recommends the use of paracetamol and the topical application of non-steroidal anti-inflammatory

Figure 5 Frequency of the pharmacons according to relevance with the disorder in question

Distribution of the used pharmacons according to ATC categories

- A02B: Drugs for peptic ulcer and gastro-oesophageal reflux disease (GORD)

- M01-02-03 Anti-inflammatory and antirheumatic products, Topical products for joint and muscular pain, Muscle relaxants

- N02: Analgesics

Regarding to the Figure is demonstrated the number of medicine boxes diveded by years. The pharmacons were counted based on the patients documentation. The mentioned ATC categories (A02B, M01-02-03, N02) were selected in accordance with NICE guideline therapeutic suggestion.

Non-pharmacological therapies were very diverse, all the options in modern medicine were available for the patients. The percentage distribution of the treatments shows that the rates of gymnastic exercises and massage were the highest. Patients received these in $\sim 60 \%$ and $\sim 70 \%$ of the cases. When discharged, the patients were recommended to continue gymnastic exercises at home on a daily basis; the nature of the exercises was such that after training the patients could perform them on their own, without continuous expert su-

drugs (NSAIDs) as first-line agents; followed by oral NSAIDs as the next step, and finally weak opiates (tramadol and its derivatives); the latter is frequently the first-line therapy for the elderly. Given that paracetamol and the vast majority of NSAID formulations are available without a medical prescription and the diagnosis is not established, the proportion of formulations purchased specifically for musculoskeletal complaints cannot be determined without a special questionnaire survey. Furthermore, the evaluation is limited by the fact that this active ingredient group includes hundreds of active ingredients and there is no clear recommendation for a particular one. Selective inhibitors for cyclooxygenase type 2 enzyme are mostly prescription-only preparations and can thus be monitored. It must be mentioned that their excessive or unjustified use may result in cardiovascular complaints.

The active ingredient diclofenac, which is not selective for the enzyme type, is also the active ingredient in a great number of preparations for musculoskeletal complaints; in this case, many non-prescription drugs are available, and their use for this purpose cannot be assessed without a special survey. It should be noted here that the prolonged and combined use of non-selective NSAIDs may result in the development of severe gastrointestinal adverse effects or in the exacerbation of the existing symptoms; patient education is essential in this stage as well.

Based on the final discharge reports, , a total of 3,313 drugs were administered to 400 patients, which means an average of $\sim 8$ drugs per patient. Recorded NSAIDs, formulations containing paracetamol and tramadol were included in the evaluation; and also proton pump inhibitors, which the recommendation strongly recommends 


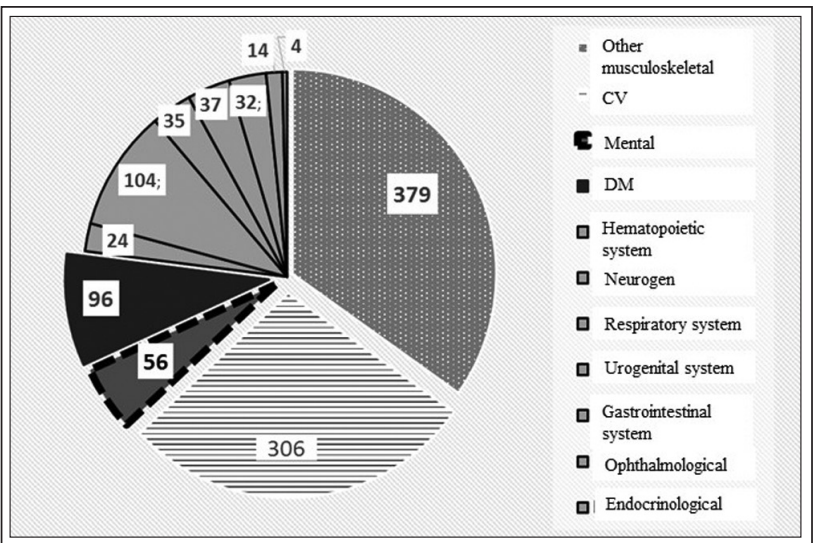

Figure 6 Incidence of co-morbidities during the period studied

for use with NSAIDs. The drugs used were analyzed with the consideration of the ATC codes of the active ingredients, the results are summarized in Figure 5.

It is noteworthy that first-line paracetamol was mentioned only in 11 cases, while tramadol and its derivatives were taken in 168 cases (group $\mathrm{NO} 2$ ), which had been expected considering the age distribution (grey line). Another important finding is that despite taking a great amount of non-steroids, the number of gastric protectors is negligible (NSAID - black line, PPI - proton pump inhibitors - broken line). The curves of non-steroids and PPIs run essentially parallel to each other in the period studied, which suggests that in some cases PPI was used while taking NSAIDs, but generally only in one third of the cases.

\subsection{Existing co-morbidities}

Existing co-morbidities were also recorded in the patients' final reports and their evaluation is shown in Figure 6. According to the data, the diagnosis contained "other musculoskeletal complaint" almost without exception, which means 379 cases (grey dots). Some cardiovascular disease occurred in $70-80 \%$ of the cases in the test sample (black stripes). DM was also present consistently each year with an incidence of 20-30\% (black marking), and the basic disease was accompanied by mental/psychological problems in many cases (broken lines).

\section{Discussion}

In conclusion, as a result of aging societies, arthrosis will result in an increasing number of patients worldwide, thus in Hungary as well. The situation may be worsened by low health literacy, the general lack of health-conscious behavior, and unhealthy and sedentary lifestyles, which are common problems in today's societies.

It is a particularly great challenge to make the diagnosis in time as the disease is "silent" for a while; pain and impaired mobility that interfere with daily activities occur only in the advanced stage of the disease. By this time, arthrosis is of such extent that the affected population needs continuous treatment.

The knowledge of the factors leading to the development of arthrosis and the analysis of the available patient documentation are absolutely necessary to establish the Hungarian population's state and, on the basis of the facts, to explore potential intervention points in order to improve the patients' quality of life.

The current survey, based on the international guideline, processes and evaluates data from a representative sample of Hungarian patients, in the groups of the recommended therapies, broken down according to non-pharmacological and pharmacological therapies.

A significant proportion of the Hungarian patient population selected by randomized sampling is in active working age, and the majority of them belong to the overweight or obese categories.

Non-pharmacological rehabilitation treatments are widespread, they are efficient and result in a long-term asymptomatic period; however, the patients discharged from the treatment have to do the exercises recommended by doctors and physiotherapists on their own at home every day in order to maintain the achieved results, so the patients' conscious adherence is essential.

Pharmacological therapy is characterized mainly by the use of over-the-counter preparations, which the patients commonly take in excess and in combination, parallel to their prescription-only counterparts. This phenomenon may induce adverse effects, the development or worsening of comorbidities. Irrespective of this, co-morbidities affecting the cardiovascular and metabolic systems are more common in arthrosis, which was highlighted in our study.

\section{Conclusion}

In order to comply with the guideline on patient education, the multidisciplinary collaboration of Hungarian healthcare professionals working in different areas is required. Besides family doctors, 
specialists and physiotherapists, pharmacists also have a prominent role due to the phenomena experienced in pharmacological therapy. Pharmacists taking part in the practice, in collaboration with dietitian and physiotherapist colleagues, can ensure mutual and ongoing communication by providing relevant information to the doctor responsible for therapy. In this way, patient care is shared by professionals with appropriate competences, the excessive use of unnecessary drugs taken on the patient's own initiative can be avoided, the monitoring of recommendations for lifestyle and exercises at home can be shared, and adherence can be maintained longer. The simultaneous and combined use of all these, the collaboration of healthcare professionals is the key to successful therapeutic outcome and the resulting improvement in the quality of life in the case of osteoarthrosis as well.

\section{References}

1. World Health Organisation, Adherence to long term therapies - Evidence for action, available from: http://www.who.int/chp/knowledge/publications/ adherence_report/en/ [Accessed: November 15, 2016], 2003, Chapter V.

2. Stoffer MA, Smolen JS, Woolf A et al. Development of patient-centred standards of care for osteoarthritis in Europe: the eumusc.net-project Ann. Rheum. Dis. 2015;74: 1145-1149. https://doi.org/10.1136/annrheumdis-2014-206176

3. Jordan KM, Arden NK, Doherty $M$ et al. EULAR Recommendations 2003: An Evidence based approach to the management of knee osteoarthritis: Report of a Task Force of the Standing Committee for International Clinical Studies Including Therapeutic Trials (ESCISIT) Ann Rheum Dis. 2003;62: 1145-1155. https://doi.org/10.1136/ard.2003.011742

4. EUROSTAT Musculoskeletal Health In Europe Report V5.0 Available from: https://www.google. $\mathrm{hu} / \mathrm{search}$ ?q=eumusc .net $\& \mathrm{ie}=u t f-8 \& o e=u t f-$ $8 \& c l i e n t=$ firefox $-b \& g f e \_r d=c r \& e i=01 w t W b O H L I q B$ X8PMvbAH\#q=eumusc.net+report+v5.0 [Accessed: October 10, 2016]

5. Bone and Joint Decade, Global Alliance of Musculoskelatal Health, Available from: http://bjdonline.org/ [Accessed: November 30, 2016]

6. Csont és Ízület évtizede (2010-2020) Magyarországon, Available from: http://www.sulypont.hu/ blog/gyogytestneveles-kategoria/csont-es-izulet- evtizede-alapitvany-2010-2020-magyarorszagon [Accessed: November 30, 2016]

7. Allen KD, Golightly YM Epidemiology of osteoarthritis: state of evidence. Curr. Opin. Rheumatol. 2015; 27(3): 276-283. https://doi.org/10.1097/ BOR.0000000000000161

8. Palazzo C, Nguyen C, Lefevre-Colau MM et al. Risk factors and burden of osteoarthritis Ann. Phys. Rehab. Med. 2016; 59(3): 134-138. https://doi.org/10.1016/j. rehab.2016.01.006

9. Silverwood V, Blagojevic-Bucknall M, Jinks C et al. Current evidence on risk factors for knee osteoarthritis in older adults: a systematic review and metaanalysis, Osteoarthritis and Cartilage 2015; 23(4): 507-515. https://doi.org/10.1016/j.joca.2014.11.019

10. Hungarian Central Statistical Office European Health Interview Survey, 2014 [Központi Statisztikai Hivatal (KSH) Európai Lakossági Egészségfelmérés, 2014] Available from: http://www.ksh.hu/apps/shop. kiadvany?p_kiadvany_id=79467\&p_lang=HU [Accessed: December 15, 2016] [Hungarian]

11. European Medicines Agency (EMA) guideline on clinical investigation of medicinal products used in the treatment of osteoarthritis, London, January 20, 2010.

12. Lockwood W Osteoarthritis (Degenerative Joint Disease), Available from: http://www.rn.org/courses/coursematerial-247.pdf [Accessed: November 15, 2016]

13. Fernandes L, Hagen KB, Bijlsma JWJ et al. EULAR recommendations for the non-pharmacological core management of hip and knee osteoarthritis Ann. Rheum. Dis. 2013; 0: 1-11

14. National Institute for Health and Care Excellence (NICE) Rheumatoid arthritis in adults: management, Available from: https://www.nice.org.uk/guidance/ng100 [Accessed: October 24, 2018]

15. National Institute for Health and Care Excellence (NICE) Osteoporosis All NICE products on osteoporosis. Includes any guidance, advice, NICE Pathways and quality standards. Available from: https:// www.nice.org.uk/guidance/conditions-and-diseases/diabetes-and-other-endocrinal--nutritional-andmetabolic-conditions/osteoporosis\#panel-pathways [Accessed: October 24, 2018]

16. National Institute for Health and Care Excellence (NICE) Osteoarthritis: care and management, Available from: https://www.nice.org.uk/guidance/cg177 [Accessed: December 15, 2016]

17. Veronese N. Adherence to mediterranean diet is associated with lower incidence of frailty: DATA from the osteoarthritis initiative, World Congress on Osteoporosis Osteoarhritis and Musculoskeletal disorders, Florence Italy, 23-26 March 2017 